Num 20 - Julio-Diciembre, 2015 (Publicación preliminar)

\title{
El trastorno de estrés postraumático en niños preescolares: una revisión literaria
}

\section{Post-traumatic Stress Disorder in Preschooler's Children: a literary review}

\author{
Kelly Romero Acosta* \\ Federico Ruiz ${ }^{* *}$
}

\section{Resumen}

El desarrollo de psicopatologías en preescolares es un tema reciente de estudio. Varios grupos de investigadores se han tomado la tarea de investigar este fenómeno, y han encontrado interesantes hallazgos que dejan al manual diagnóstico y estadístico de los trastornos mentales (DSM) y a los instrumentos de media como inadecuados para valorar con precisión las psicopatologías presentes en la infancia temprana. Por esta razón, el DSM-5 trae consigo nuevos criterios diagnósticos pensados para niños menores de cinco años. El trastorno de estrés postraumático se caracteriza por aparecer posterior a la vivencia de acontecimientos estresantes. Los niños pre-escolares no son la excepción. El propósito de este escrito es realizar una actualización sobre los últimos hallazgos en la investigación del trastorno de estrés postraumático en niños preescolares. Al final del texto se lleva a cabo una reflexión sobre las implicaciones de estos descubrimientos en la clínica.

Palabras clave: trastorno de estrés postraumático, niños preescolares, trauma y evaluación.

\footnotetext{
* Kelly Romero-Acosta es doctora y magister en Psicología Clínica y de la Salud de la Universidad Autónoma de Barcelona (España). Correo electrónico kromero@ unimetro.edu.co.

${ }^{* * *}$ Federico Ruiz, estudiante de X semestre de Psicología de la Universidad Metropolitana de Barranquilla.
} 


\begin{abstract}
Psychopathology in preschool age has been researched in the recent years. Several groups of researchers have explored this phenomenon and they have found interesting findings which determines the DSM IV and the psychological instruments as inadequate to value psychopathology in early childhood. For this reason, the DSM-V is implementing new diagnostic criteria designed especially for children under five years old. The Posttraumatic Stress Disorder is characterized by arising after events that produce high levels of distress; and pre-schoolers are not the exception. The aim of this paper is to perform an update of the latest findings about the Posttraumatic Stress Disorder in young children. At the end of the text, a reflection on the implication of the research findings in clinical field is accomplished.
\end{abstract}

Key Words: Posttraumatic stress disorder, young children, trauma, preschool y Assessing.

\title{
Introducción
}

El Manual diagnóstico y estadístico de los trastornos mentales (DSM-IV, APA, 1994), en su cuarta versión, señala por primera vez que los niños pueden sufrir, al igual que los 
Num 20 - Julio-Diciembre, 2015 (Publicación preliminar)

adultos, por secuelas de eventos traumáticos. No obstante, en esta versión se habla muy poco de las características de este trastorno en niños pequeños, puesto que se creía que por la falta de desarrollo no podían sufrir psicopatologías.

En la actualidad se realizan importantes esfuerzos para demostrar que los niños preescolares pueden presentar psicopatologías propias hasta ahora de niños mayores o adultos (Research Diagnostic Criteria - Preschool Age, RDC-PA, 2002). El DSM-V ha tenido en cuenta estos hallazgos y en su nueva versión apuesta por detallar criterios diagnósticos específicos para niños menores de cinco años.

Sheeringa y sus colaboradores (2001) aseguran que en los tres primeros años de vida la psicopatología es expresada y experimentada por los niños a pesar de un menor grado de desarrollo cognitivo, perceptual y lingüístico. Esto quiere decir que los niños preverbales pueden sufrir un Trastorno por Estrés Post-Traumático (TEPT) severo, aunque no hablen bien. Ocho de los diecinueve síntomas especificados en los criterios diagnósticos del TEPT en el DSM-IV necesitaban un informe verbalizado, lo que no se adecuaba a la forma de manifestación sintomática de los más pequeños.

Ahora bien, el problema se incrementa con la falta de consenso que existe en la concepción de la presentación de los cuadros psicopatológicos en las diferentes edades, asimilando cambios normativos en el desarrollo. Actualmente se puede usar como sistema de clasificación diagnóstica el DSM-5 (APA, 2013), el DSM IV-TR (American Psychiatric Association, 2001) y La CIE-10 (World Health organization, 1992). Especialmente para niños preescolares, la organización Zero to Three ha desarrollado la Diagnostic Classification of Mental Health and Developmental Disorders of infancy and Early Childhood (DC: 0-3, 1994) como un intento para llenar los vacíos dejados por el DSM-IV 
(APA, 1994). Esta incluye puntos de los sistemas anteriores, medidas diagnósticas adaptadas a niños preescolares, nuevas consideraciones y, también, nuevos cuadros.

El diagnóstico y la evaluación de niños de 0 a 5 años pueden ser complejos en tanto se evidencian constantes cambios en su desarrollo. Las manifestaciones de perturbaciones clínicas pueden ser menos diferenciadas o, incluso, cambiar a través del tiempo en respuesta a la maduración. El impacto que provoque el trauma en el niño depende de su desarrollo, principalmente cognitivo y emocional (Yule \& Williams, 1990).

Hay evidencia que demuestra que los niños pequeños expuestos a hechos violentos pueden salir psicológicamente afectados. Linares y colaboradores, en el 2001, encontraron que el estrés maternal puede mediar la relación entre la exposición a hechos violentos y el desarrollo de problemas comportamentales en niños de tres a cinco años de edad. Estos niños vivían en vecindarios con altos niveles de violencia y el $42 \%$ de los niños de la muestra había sido testigo de al menos un hecho violento.

Colombia es un país con altos niveles de violencia no solo en el contexto urbano sino también rural. Cuevas \& Castro (2009) examinaron la relación entre niveles de exposición de violencia directa e indirecta y los efectos emocionales (ansiedad y depresión) y conductuales en una muestra de 1362 niños de entre los 7 y 14 años radicados en la ciudad de Cali (Colombia). Ellos hallaron diferencias significativas entre niveles de exposición a hechos violentos y la presentación de ansiedad, depresión, estrés postraumático, agresión y delincuencia.

El siguiente artículo tiene como objetivo actualizar al lector sobre la presentación del TEPT en niños preescolares. En primer lugar se detallarán los criterios diagnósticos del 
TEPT en la DC: 0 a 3 años y las implicaciones fisiológicas del cuadro. También, se abordará el papel de los cuidadores y los factores incrementadores del TEPT. Por último, se enumerarán los instrumentos de evaluación para niños preescolares y se analizarán los tratamientos.

\section{Método}

Se ha efectuado la búsqueda de información a partir de la base de datos PsycINFO, de la hemeroteca y biblioteca de humanidades de la Universidad Autónoma de Barcelona (UAB) UAB y de PubMED, con las palabras clave: posttraumatic stress disorder, young children y preeschool.

\section{Resultados}

\section{El Trastorno de Estrés Postraumático en la DC: 0-3}

Según el DSM IV (1994) el trastorno de estrés postraumático se caracteriza por la aparición de síntomas que siguen a la exposición de un acontecimiento estresante y en extremo traumático en el que el individuo se ve envuelto en hechos que representan peligro real para su vida o cualquier otra amenaza para su integridad física. También puede ocurrir que el individuo sea testigo de un hecho perturbador donde se producen muertes, daño serio, peligro de muerte o heridas graves.

Ahora bien, las manifestaciones sintomáticas especificadas desde el criterio A1 no siempre reflejan la manera particular de manifestación de los niños preescolares; por ello, gracias a las últimas investigaciones se ha podido conocer cómo los niños más pequeños 
pueden expresar los síntomas de estrés posteriores a un trauma sin necesariamente cumplir todos los criterios del DSM (APA, 2013, 1994; Scheeringa, et al., 2003). La tabla 1 muestra una comparación de los criterios diagnósticos del DSM-IV, del DSM-V y de la DC- 0 to 3.

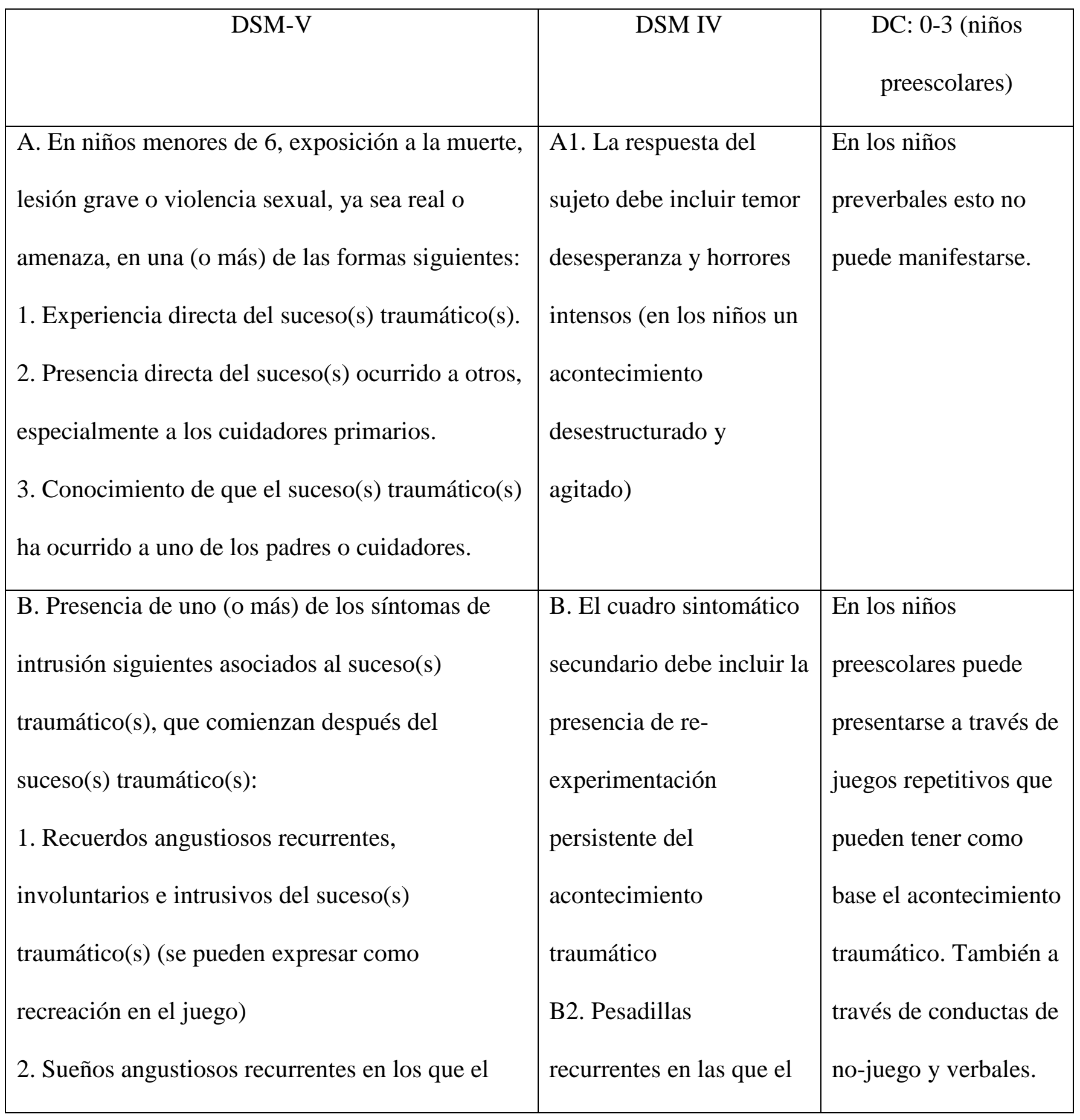


Num 20 - Julio-Diciembre, 2015 (Publicación preliminar)

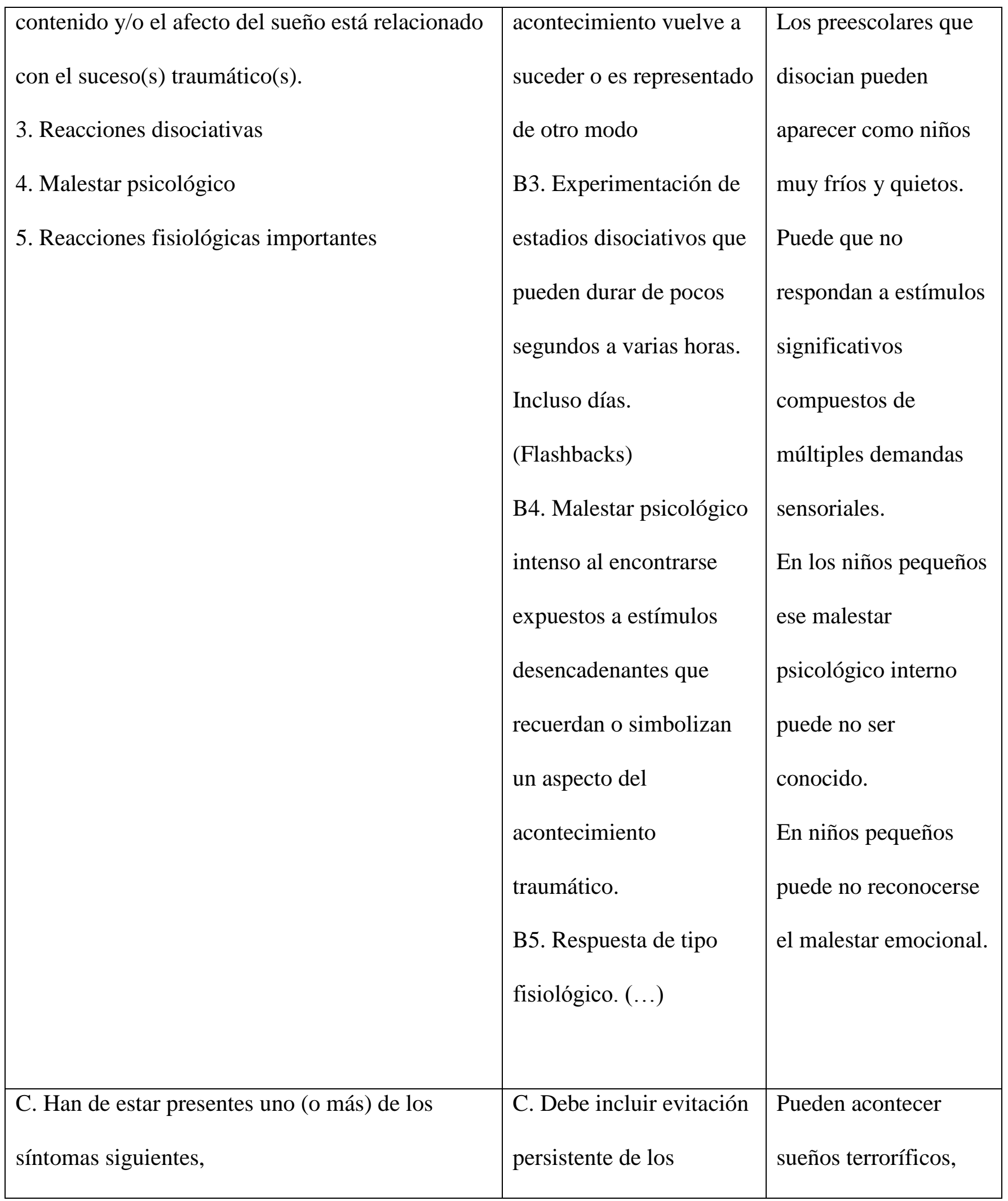


Num 20 - Julio-Diciembre, 2015 (Publicación preliminar)

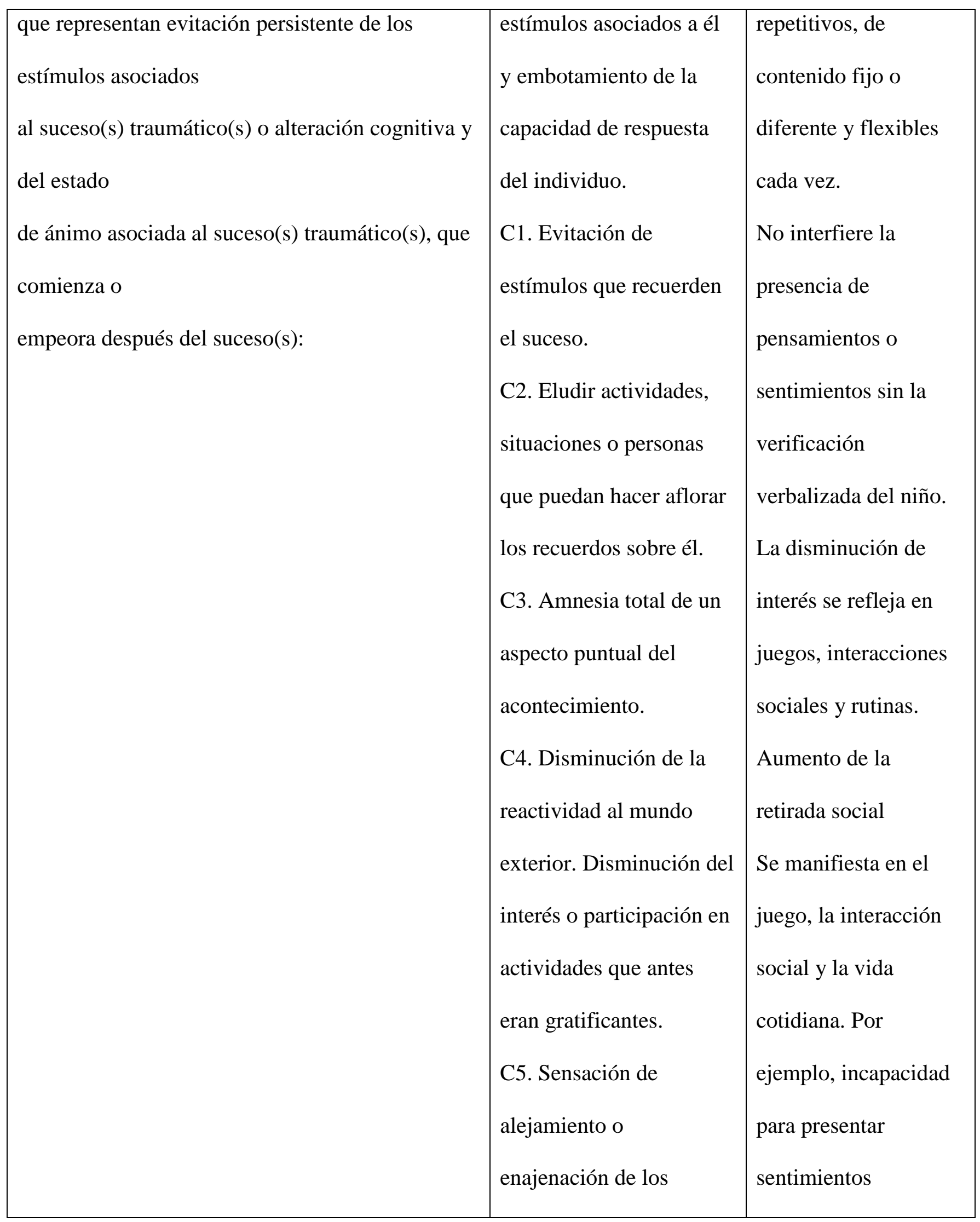


Num 20 - Julio-Diciembre, 2015 (Publicación preliminar)

\begin{tabular}{|c|c|c|}
\hline & $\begin{array}{l}\text { demás. } \\
\text { C6. Disminución de la } \\
\text { necesidad para sentir } \\
\text { emociones. } \\
\text { C7. Sensación de futuro } \\
\text { limitado. }\end{array}$ & amorosos. \\
\hline D. Alteración importante de la alerta y reactividad & D1. Debe incluir & Incremento en la \\
\hline asociada al suceso(s) traumático(s), que comienza & síntomas presentes de & dificultad para \\
\hline o empeora después del suceso(s) traumático(s), & activación arousal. & conciliar o mantener \\
\hline como se pone de manifiesto por dos (o más) de las & Ansiedad constante que & el sueño. Protesta para \\
\hline características siguientes: & no existía antes del & ir a la cama. \\
\hline 1. Comportamiento irritable y arrebatos de furia & trauma y/o dificultad & Incremento de \\
\hline (con poca o ninguna provocación) que se expresa & para conciliar o mantener & irritabilidad o ataques \\
\hline típicamente como agresión verbal o física contra & el sueño. & de ira \\
\hline personas u objetos (incluidas pataletas extremas). & D2. Irritabilidad o & Incremento de la \\
\hline 2. Hipervigilancia. & ataques de ira & dificultad de \\
\hline 3. Respuesta de sobresalto exagerada. & D3. Dificultad para & concentración \\
\hline 4. Problemas con concentración. & concentrarse o ejecutar & \\
\hline 5. Alteración del sueño (p. ej., dificultad para & tareas. & \\
\hline conciliar o continuar el sueño, o sueño inquieto). & $\begin{array}{l}\text { D4. Hipervigilancia } \\
\text { D5. Respuestas } \\
\text { exageradas de sobresalto }\end{array}$ & \\
\hline
\end{tabular}




\begin{tabular}{|l|l|l|}
\hline E. La duración de la alteración es superior a un & El cuadro sintomático & \\
mes. & completo debe estar & \\
presente más de un mes. & (Criterio E) & \\
significativo o problemas & Provoca un malestar & \\
en la relación con los padres, hermanos, & clínicamente & \\
compañeros u & significativo o deterioro & \\
otros cuidadores, o en el comportamiento en la & áreas importantes de la & \\
escuela. & vida del individuo. & \\
fisiológicos de una sustancia (p. ej., medicamento & & \\
o alcohol) u otra afección médica. & & \\
\hline G. La alteración no se puede atribuir a los efectos & & \\
\hline
\end{tabular}

La Research Diagnostic Criteria - Preschool Age (RDC-PA, 2002) enumera algunos síntomas específicos en niños preescolares tales como, terrores nocturnos, agresiones nuevas, ansiedad por separación marcada, nuevos miedos sin relación con el trauma (miedo a ir a la baño o a la oscuridad), nueva conducta desafiante, conductas repetitivas sin afecto, pérdida de habilidades adquiridas (control de esfínteres, pérdida del habla...), entre otros.

\section{Implicaciones fisiológicas del cuadro}


Se han hallado implicaciones fisiológicas debidas al TEPT, sin embargo, aún no está claro si son resultado de TEPT directamente, si están relacionadas a factores predisponentes o si son resultado de problemas comórbidos (Scheeringa et al., 2004). Los hallazgos incluyen:

- Volumen del hipocampo más pequeño en individuos con TEPT

- Áreas del cerebro comprometidas en la percepción de amenaza (por ejemplo, la amígdala) han alterado el metabolismo en sobrevivientes adultos del trauma

- La actividad del cingulado anterior (en áreas del cerebro que inhiben la amígdala y otras regiones cerebrales implicadas en la respuesta de miedo) decrece en personas con TEPT.

- Bajos niveles de cortisol basal.

- Concentración de receptores de glucocorticoide y, posiblemente, incremento de la actividad en el hipocampo.

También, en una investigación sobre niños quemados (Stoddard, Saxe, Rondefeldt, Drake \& Burns et al., 2006) se encontró que estos aparecían con síntomas agudos de tensión, Sheeringa, y sus colegas (2005), encontraron que las experiencias traumáticas en niños preescolares pueden dar lugar a cambios profundos en el neurodesarrollo. En su investigación los niños traumatizados disminuyeron el ritmo cardíaco en respuesta a un estímulo traumático.

\section{EI TEPT y su relación con el papel de los cuidadores}


Num 20 - Julio-Diciembre, 2015 (Publicación preliminar)

Los trastornos tempranos en la niñez perjudican, persisten y se asocian con factores de riesgos psicopatológicos (por ejemplo: la pobreza, la familia, la historia psiquiátrica; Briggs-Gowan, Carter, Skuban, \& Horwitz, 2001; Briggs-Gowan et al., 2003; Egger \& Angold, 2006b; Egger et al., 2006a; Dyregrov \& Yule, 2006; Keenan, Shaw, Walsh, Delliquadri, \& Giovannelli, 1997; Lavigne et al., 1998). La influencia de los padres, en especial de la madre, en el desarrollo o no desarrollo de psicopatologías ha sido demostrada a través de investigaciones realizadas en los últimos años. Scheeringa y colaboradores, en 1995, encontraron que cuando la amenaza o el hecho traumático implican al cuidador existe más probabilidad de desarrollar TEPT.

El "soporte maternal” (Belden \& Luby, 2006) se puede conceptualizar como el grado de consideración positiva y acercamiento de las madres a los hijos de tal manera que facilite la sensación de autonomía y favorezca los intentos de aquellos para crear estrategias con el fin de resolver problemas. Denham y Grout (1993) informaron que los hijos de las madres que responden óptimamente a las expresiones emocionales de sus hijos -incluidas rabia, tristeza, felicidad y miedo- son capaces de reproducir estas actitudes y sentimientos en ausencia de sus cuidadores. Igualmente Eisenberg y colegas (1992) demostraron que los padres que responden a sus hijos con críticas y emociones de tristeza, hacen de ellos niños menos simpáticos y más propensos a la rabia y a las emociones negativas.

En efecto, existe evidencia empírica de la importancia que tiene la relación cuidador-niño en el desarrollo, mantenimiento y cura del trastorno, especialmente en la infancia temprana, cuando la dependencia del niño a la madre es mucho mayor (Chu \& Lieberman, 2010; Dyregrov \& Yule, 2006; Gunnar \& Cheatham, 2007; Linares, Heeren, Bronfman, Zuckerman, Augustyn, et al., 2001; Lynch \& Cicchetti, 1998). Se ha encontrado 
relación entre soporte maternal, mal funcionamiento familiar, o ambas, y los trastornos de los niños, situaciones más frecuentes y fuertes mientras más pequeño es el preescolar, es decir, de 3 a 4 años de edad (Laor, Wolmer \& Cohen, 2001; Chu \& Lieberman, 2010). Sheeringa y sus colegas (2002) encontraron que se presenta un efecto significativo entre la disciplina positiva parental, síntomas de TEPT y ritmo de respiración. Los niños más comprometidos disminuyeron el ritmo de la respiración cuando hubo menos refuerzo positivo por parte de los cuidadores.

En ocasiones, puede que la madre haya estado implicada en el suceso traumático pero el niño no, produciendo, a pesar de ello, efectos traumáticos en el niño (Scheeringa, et al., 2005; Dyregrov \& Yule, 2006; Lieberman, Van Horn \& Ozer, 2005).

Una de las variables más predictivas de sintomatología severa es la que implica a los cuidadores en el evento (Sheeringa et al., 1995). Esta implicación puede ser entendida de dos maneras: 1) puede ser que el niño presenció las implicaciones de una amenaza sobre el cuidador o, 2) puede ser que el cuidador y el niño pudieron estar implicados al mismo tiempo en la amenaza. En ambas situaciones se suele presentar un incremento de la sintomatología debido a una inadecuada respuesta emocional del cuidador implicado hacia el pequeño.

La relación de la madre con el niño depende de la habilidad de ella para leer y responder acertadamente a las necesidades del pequeño, esto es, los efectos de esta relación como fortaleza de un acontecimiento traumático pueden ser tales que moderen la intensidad de síntomas que genere el evento traumático (Chu \& Lieberman, 2010; Dyregrov \& Yule, 2006; Linares, et al., 2001; Quota, Punamaki \& El Sarraj, 2003). Dicho de otro modo, si en 
medio del trauma, la madre es capaz de responder con tranquilidad y seguridad a las necesidades del niño, esto puede moderar la intensidad de los futuros síntomas de TEPT.

También se puede dar el caso de que tanto la madre como el niño estén traumatizados. En estas situaciones ambos pueden llegar a afectarse mutuamente y producir síntomas que pueden ir de moderados a severos dependiendo de la relación con el cuidador (Chu \& Lieberman, 2010).

\section{Factores que influyen en el incremento del TEPT}

Diversos estudios han señalado algunos factores que pueden influir en el incremento de la sintomatología del TEPT (Chu \& Lieberman, 2010; Stoddard, et al., 2006), entre ellos se encuentran: poco soporte parental, compromiso de los cuidadores en la amenaza, mayor exposición del trauma en incidentes traumáticos, preexistencia de trastorno psiquiátrico, varias exposiciones al trauma, trauma causado por personas, especialmente por cuidadores, ansiedad de los cuidadores, intensidad del dolor, síntomas de los padres, magnitud del trauma, entre otros.

Stoddard y colaboradores (2006) estudiaron los efectos estresantes de diferentes tipos de quemaduras en los niños. Ellos hallaron que los niños con mayor estrés eran aquellos con quemaduras más graves y cuya intensidad del trauma había sido mayor. La sintomatología de los padres también afectó la presentación del trauma en los niños.

\section{La evaluación del Trastorno de Estrés Postrumático en preescolares. El modelo de Claire Pebbles (1997).}


Claire Pebbles (1997) muestra un modelo de evaluación y de tratamiento para la psicopatología en preescolares. Argumenta que el trabajo terapéutico con niños debe contar con un equipo multidisciplinario conformado por psiquiatras, psicólogos, trabajadores sociales y terapistas ocupacionales, de ser posible.

Se debe contar principalmente con la familia, profesores y cuidadores del pequeño puesto que ellos son la pieza fundamental para entender lo que acontece en vista de la ausencia de desarrollo verbal y cognitivo. Por ello, es primordial una historia clínica muy completa. La evaluación puede durar entre cuatro a cinco horas incluyendo una serie de entrevistas estructuradas que tratan la situación psiquiátrica del niño y el soporte parental. La relación entre el apego y las experiencias traumáticas deben convertirse en un componente integral en la evaluación y el tratamiento en niños preescolares (Chu \& Lieberman, 2010). En este sentido, es de vital importancia trabajar conjuntamente con el cuidador o cuidadores del preescolar.

Posterior a las evaluaciones, el cuidador, el niño y el clínico entran a un cuarto de juego dotado de elementos lúdicos apropiados para la edad del paciente que puedan ayudar a realizar una serie de actividades estructuradas y libres, filmadas y vistas a través de un espejo por el resto del equipo. La sesión de juego puede demorar de 30 a 45 minutos y se divide en tres segmentos: los primeros diez minutos incluyen un juego libre no estructurado que permiten al niño explorar el espacio y lograr empatía con el entrevistador. Luego, se propicia que el cuidador y el niño jueguen con el fin de observar su relación: ¿qué tan autónomo es el niño? ¿El padre se presenta como soporte? ¿Está atento a las preguntas y necesidades del pequeño? ¿Las aborda correctamente? ¿Qué dinámica emocional existe entre los dos? Finalmente, el clínico se hace partícipe del juego para conocer el nivel de 
desarrollo del niño (gestos, comunicación verbal, nivel de actividad motora, nivel de atención, humor, entre otras).

\section{Instrumentos de evaluación del TEPT en preescolares}

Scheeringa y sus colegas (2005) realizaron un estudio longitudinal con niños preescolares y encontraron que en la primera visita se predijeron significativamente más síntomas y daño funcional que durante los dos años siguientes al estudio, sin embargo, estos no fueron desapareciendo a través del tiempo, como en los adultos, sino que, más bien, se presentaban doblemente más vulnerables a remisiones. Esto hace pensar sobre un posible compromiso neurobiológico a partir de experiencias traumáticas. En esta medida se hace indispensable estructurar instrumentos de evaluación que identifiquen la potencia del trauma en el preescolar y, al mismo tiempo, consiga diferenciar características inherentes del trauma de cambios en el desarrollo. Ahora bien, ¿con qué instrumentos se cuenta para el trastorno del estrés postraumático en preescolares?

La creación de instrumentos de medida para niños preescolares implica ciertos requisitos específicos. En primer lugar, la mejor manera de formular preguntas a los pequeños es aquella cuya respuesta suscita un SÍ o un NO (Stover \& Berkowitz, 2005). Así mismo se considera de vital importancia la inclusión del juego para realizar las evaluaciones. Los siguientes son instrumentos de evaluación del TEPT para niños preescolares.

Trauma Symtom Checklist for Young Children. 
La Truma Symptoms Checklist for Young Children (TSCYC, Briere, Jonson, Bisada, Damoon, Crouch et al., 2001) es una escala diseñada para niños de edades comprendidas entre 3 y 12 años de edad en forma de checklist. Contiene ocho escalas clínicas, todas con referencia al estrés: intrusión de estrés post-traumático, evitación de estrés post-traumático, nivel de arousal de estrés post-traumático, preocupaciones sexuales, disociación, ansiedad, depresión y rabia-agresión. Además contiene una sumatoria total de las escalas.

\section{Posttraumatic Stress Disorder Semi-Structured Interview and Observation Record.}

Se basa en una entrevista semiestructurada suministrada a padres e infantes (Scheeringa \& Zeanah, 1994). En primer lugar, se le pregunta al padre del niño acerca de posibles traumas que el pequeño ha podido sufrir, para luego profundizar sobre los síntomas. Los padres pueden hablar sobre el inicio, la frecuencia y la duración de los síntomas. Se basa en el DSM IV (APA, 1994) y en la DC 0-3 (1994). Esta entrevista requiere un alto nivel de experticia clínica puesto que el entrevistador debe distinguir los síntomas en el niño a partir de SÍ y NO.

\section{Postraumatic Symtom Inventory for Children}

La Postraumatic Symtom Inventory for Children (PTSIC; Eisen, 1997) se administra a niños de entre cuatro y ocho años de edad y se basa en los criterios B, C y D del DSM IV (APA, 1994). Tiene en cuenta doce eventos traumáticos, entre los que se encuentran accidente de carro, abuso sexual, ser el testigo o la víctima de una acción violenta, entre otros. El test se encuentra diseñado de tal manera que si al preguntar un ítem el niño contesta negativo, se puntúa con " 0 " y se sigue a la siguiente pregunta. Ahora bien, si 
responde afirmativamente se formulan los interrogantes que siguen al ítem para conocer la frecuencia. Algunas veces se puntúa con "1" y otras con “2”, la puntuación total es de 60, dato que refleja la frecuencia de los síntomas.

\section{Modified Semi-Structure Interview and PTSD Reaction Index}

Esta entrevista se basa en la observación directa y el juego, y se inicia con la realización de un dibujo por parte del niño (Pynoos \& Nader, 1986). Luego prosiguen entre veinte y treinta minutos de juego libre. El pequeño tendría que responder a una serie de preguntas con "sí", "no" o "no sé". Cabe destacar que toda la sesión es filmada. La entrevista no proporciona códigos de interpretación ni para el dibujo, ni para la observación, así que todo se basa en la experticia del terapeuta.

\section{Preschool Aged Psychiatric Assesssment}

La Preschool Aged Psychiatric Assesssment (PAPA, Egger, Angold, Ascher, 1999) es una entrevista que proporciona definiciones de síntomas, el entrevistador puede llegar a ellos a partir de preguntas. Los entrevistadores codifican la intensidad, frecuencia, duración y fecha de inicio, enfocándose principalmente en los tres últimos meses. Está basada en la Child and Adolescent Psychiatric Assessment (CAPA, Angold, Prendergast, Cox, Harrington, Simonoff et al., 1995) aplicable a niños de 5 a 18 años de edad. Se desarrolló con base en los criterios del DSM IV, la CIE-10 y la DC: 0-3. Igualmente, se añaden ítems relevantes de la Child Behavior Checklist (CBCL, Achenbach \& Edelbrok, 1983; Achenbach \& Rascorla, 2000) así como revisiones de otras medidas diagnósticas para preescolares que no cubre la CAPA. Se revisó literatura de psicopatología preescolar e 
Num 20 - Julio-Diciembre, 2015 (Publicación preliminar)

incluyó síntomas y comportamientos fruto de investigaciones como criterios alternativos para el estrés postraumático.

\section{Acerca del tratamiento}

Claire Pebbles (1997) proporciona un modelo de tratamiento para trabajar con preescolares. El tratamiento se centra en la familia como un todo que actúa como base para ayudar al niño; por tanto, las metas de intervención subyacentes se estructuran en relación con el problema que acarrea la sintomatología del niño en la familia. Las intervenciones propenden a ser cognitivas, comportamentales y psicodinámicas, enfocadas en el niño y en el cuidador. Se puede llegar a ofrecer terapia interaccional padre-niño, manejo de comportamiento, manejo de medicación o terapia individual o en grupo, dependiendo de las necesidades del niño. La terapia interaccional se enfoca en ayudar tanto al niño como al cuidador a realizar conexiones positivas y a aprender a responderse recíprocamente.

Cabe señalar que el trabajo terapéutico lo realiza conjuntamente el clínico, el cuidador y el niño en la misma sesión, utilizando el juego como el medio de conexión. La tarea del clínico es crear una atmósfera positiva en la cual la díada pueda aprender mutuamente mientras disfrutan. Asimismo, enseña al cuidador a entender las necesidades del pequeño y le indica cómo dar suficiente confianza y libertad para lograr su autonomía, a través de guías verbales, modelado y role playing.

Las sesiones para padres ofrecen educación sobre el desarrollo del niño y proporcionan una guía que específica sus necesidades. Esto ayuda a comprender los antecedentes y las consecuencias del comportamiento de los hijos, por ejemplo: aprender a comunicarse claramente con el pequeño; escuchar reflexivamente y proporcionar comandos 
efectivos, aprender a incrementar buenos comportamientos (atención positiva); decrementar un comportamiento indeseable (tiempo fuera) entre otros.

Con respecto al cuidado médico, Lubit (2005) aconseja, en primer lugar, ayudar al niño a sentirse seguro, trabajando con mucho cuidado y precisión el trauma a través del juego, pues el niño podría re experimentarlo nuevamente. Es beneficioso hacer comprender a los padres la dinámica de los síntomas teniendo en cuenta que algunos de estos son normales luego de un acontecimiento traumático.

\section{Discusión}

El reciente campo de salud mental infantil ha surgido del reconocimiento creciente de la experiencia de niños en edades preescolares afectados con problemas de salud mental, lo que garantiza la necesidad de una intervención clínica temprana (Call, Galenson, \& Tyson, 1983, 1984; Fraiberg, 1980; Minde \& Minde, 1986; Rexford, Sander, \& Shapiro, 1976). El trastorno de estrés postraumático es uno de los cuadros clínicos con más investigación en relación con la presentación en niños preescolares. Gracias al trabajo investigativo realizado hasta ahora, sobre todo del grupo de Sheeringa y sus colaboradores, se han incluido criterios diagnósticos para niños menores de seis años en la quinta versión del DSM-5 (APA, 2013).

Los niños preescolares expresan la sintomatología de los cuadros psicopatológicos de diferente manera que los niños más grandes y los adultos. Se puede manifestar la re experimentación a través de juegos repetitivos que, en ocasiones, tiene como base el acontecimiento traumático. Las pesadillas recurrentes en las que el acontecimiento vuelve a 
Num 20 - Julio-Diciembre, 2015 (Publicación preliminar)

suceder, pueden acontecer en los pequeños como sueños terroríficos, repetitivos, de contenido fijo, diferente y flexible cada vez. Así mismo, la experimentación de estados disociativos puede hacer aparecer en los preescolares estados de frialdad y quietud. También puede que no respondan a estímulos significativos compuestos de múltiples demandas sensoriales y esto puede reflejarse en juegos, interacciones sociales y rutinas. La activación arousal se presenta a través del incremento en la dificultad para conciliar o mantener el sueño y de protestas para ir a la cama. Sin embargo, otras características diagnósticas siguen igual. Por ejemplo, la amnesia total del trauma y eludir actividades, situaciones o personas que puedan hacer aflorar los recuerdos del hecho traumático, no cambian en los niños pequeños.

La investigación de la psicopatología en niños preescolares es un campo que hasta ahora se va abriendo camino, especialmente en Colombia (Romero, 2014). Los hallazgos que en los últimos años se han encontrado argumentan en gran medida la necesidad de un adecuado planteamiento de criterios diagnósticos, adaptación de instrumentos de evaluación y nuevas formas de tratamiento. Por ello, el proceso de evaluación de los preescolares debe contar con la presencia de los padres. Los cuidadores pueden contribuir a la formulación diagnóstica y a la superación del trauma, aunque, también, pueden influir en su empeoramiento. Si han estado implicados en el evento traumático o el niño les recuerda un evento traumático anterior, puede que no sean un buen soporte y apoyo para el niño. Los niños pueden desarrollar más sintomatología en ausencia de un soporte parental adecuado. Asimismo, tanto niño como cuidador pueden afectarse mutuamente si han estado ambos implicados en el trauma. Cuando el cuidador padece TEPT puede estar afectada su percepción acerca del niño. 
Num 20 - Julio-Diciembre, 2015 (Publicación preliminar)

Si un instrumento de evaluación se basa solo en el criterio del padre podrá incurrirse al sesgo de información. Los instrumentos de evaluación que tienen en cuenta las respuestas del padre, observación y respuestas del niño pueden predecir mejor el diagnóstico del TEPT. Los padres pueden suministrar información que el niño aún es incapaz de expresar por su falta de desarrollo verbal, pero el propio niño es la pieza fundamental para formular un diagnóstico preciso.

La entrevista semiestruturada de Sheeringa tiene en cuenta al niño por medio de la observación y de la formulación de preguntas sencillas que obtienen un "Sí” o un "No" como respuesta. La entrevista requiere un nivel alto de experticia por la ausencia de codificación de la observación del juego. Ahora bien, la evolución de los problemas psiquiátricos infantiles es compleja en tanto no puede incluirse el reporte de las experiencias subjetivas de los niños. Igualmente, la manifestación de los cuadros depende del desarrollo perceptual, memorístico, afectivo cognitivo y comportamental que puede variar de niño a niño dependiendo del ambiente que le circunda. En suma, los hallazgos de las investigaciones del trastorno de estrés postraumático en niños permiten hoy en día realizar un mejor trabajo en la evaluación y en la adecuación del tratamiento teniendo en cuenta todas estas variables.

En el caso de Colombia no se encuentran muchos estudios sobre la presencia de estrés postraumático en niños preescolares, aún a pesar de la alta presencia de acontecimientos vitales estresantes que pueden vivenciar tanto madre como niño en su cotidianidad debido a la situación socio-política del país. En Cali, Colombia, Cuevas \& Castro (2009) han examinado la relación entre niveles de exposición de violencia directa e indirecta y los efectos emocionales y conductuales en niños entre 7 y 14 años; sin embargo, 
no han tenido en cuenta a niños más pequeños. Se añade a esto, la falta de instrumentos de evaluación para este grupo de edad. La detección temprana de los problemas psicológicos en niños preescolares puede evitar una patología crónica o la comorbilidad de psicopatologías en el futuro. Se recomienda el artículo Assessing Violence Exposure and Trauma Symptom in Young Children: A Critical Review of Mesures de Stover \& Berkowits (2005) en tanto contiene recomendaciones para la escogencia y construcción de instrumentos de medición para niños preescolares y, también, los instrumentos que existen en el campo del trastorno de estrés postraumático y de la exposición a hechos violentos para los niños pequeños.

\section{Referencias}

Achenbach, T.M., \& Rescorla, L. (2000). Child Behaviour Checklist for Ages 11/2 - 5. Burlington. VT: University of Vermont.

Achenbach, T. M., \& Edelbrock, C. (1983). Manual for the Child Behavior Checklist and Revised Child Behavior Profile. Burlington: University of Vermont, Department of Psychiatry.

American Psychiatric Association. (2013). Diagnostic and statistical manual of mental disorders (5th ed.). Washington, DC: American Psychiatric Association (1980). Diagnostic and Statistical Manual of Mental Disorders. Tercera edición (DSM III). Washinton, DC: American Psychiatry Association. 
Num 20 - Julio-Diciembre, 2015 (Publicación preliminar)

American Psychiatric Association. (1994). Diagnostic and Statistical Manual of Mental Disorders. Cuarta edición (DSM IV). Washinton, DC: American Psychiatry Association.

American Psychiatric Association. (2001). Manual diagnóstico y estadístico de los trastornos mentales. DSM-IV TR. Barcelona: Masson.

Angold A., Prendergas M, Cox A., Harrington R., Simonogg E., Rutter M. (1995). The Child and Adolescent Psychiatric Assessment (CAPA). Department of Psychiatry, Duke University Medical Center, Durham, NC, Psychol Med., 25(4): 739-53.

Belden A., \& Luby J. (2006). Preeschoolers` Depresión Severity and Behaviors during dyadic interacitions: The mediating role of parental support. Journal of American Child and Adolescent Psychiatry, 45(2):213-22.

Briere, J., Jonson, K., Bissada, A., Damoon, L., Crouch, J., Gil, E. (2001). Trauma Symtom Checklist for Young Children (TSCYC) Reliability and association with abuse exposure in a multiñsite study. Children Abuse \& Neglect, 25, p. 1001-1014.

Briggs-Gowan, M.J, Carter, A.S, Skuban, E.M, Horwitz, S.M. (2001). Prevalence of socialemotional and behavioral problems in a community sample of 1- and 2-year old children. Journal of the American Academy of Child and Adolescent Psychiatry, 40(7):811-9.

Briggs-Gowan, M.J, Owens, P., Schwab-Stone, M., Leventhal, J., Leaf, P.J, McCue, Horowitz, S. (2003). Persistence of psychiatric disorders in pediatric settings. Journal of the American Academy Child and Adolescent Psychiatry, 42(11), 13601369. 
Num 20 - Julio-Diciembre, 2015 (Publicación preliminar)

CIE 10. (1992). Trastornos mentales y del comportamiento. Descripciones clínicas y pautas para el diagnóstico. Organización Mundial de la Salud. (ICD-10, International Classification of Diseases, 10th revision). Madrid: Mediator.

Call J, Galenson E, Tyson RL. (Ed.). (1983). Frontiers of Infant Psychiatry. Vol. 1. New York: Basic Books.

Call J, Galenson E, Tyson RL. (Ed.). (1984). Frontiers of Infant Psychiatry. Vol. 2. New York: Basic Books.

Cuevas, M.C., \& Castro, L. (2009). Efectos emocionales y conductuales de la exposición a violencia en niños y adolescentes en Colombia. Behavioral Psychology / Psicología Conductual, 17 (2), p. 277 - 297.

Chu, A. T., \& Lieberman, A. (2010). Clinical implication of traumatic stress from birth to age five. The annual review of clinical psychology, 6, 469 - 494.

Denham S, Grout L (1993) Socialization of emotion: Pathway to preschoolers'emotional and social competence. Journal of Nonverbal Behavior, 17 (3), p. 205-227.

Diagnostic Classification of Mental Health and Developmental Disorders of Infancy and Early childhood (DC: 0-3) Zero to Three. (1994). Diagnostic Classification Task Force 1994.

Dyregrov, A., \& Yule, W. (2006). A review of PTSD in children. Child and Adolescent Mental Health, 11(4),176-84.

Egger, H.L., Angold, A. (2006a). Anxiety Disorders. In: Luby J. (Ed.). Handbook of preschool mental health: Development, disorders, and treatment (137-164). New York: Guilford Press. 
Num 20 - Julio-Diciembre, 2015 (Publicación preliminar)

Egger HL, Angold A. (2006b). Common emotional and behavioral disorders in preschool children: Presentation, nosology, and epidemiology. Journal of Child Psychiatry and Psychology, 47(3/4), p. 313-337.

Egger HL, Erkanli A, Keeler G, Potts E, Walter B, Angold A. (2006c). The test-retest reliability of the Preschool Age Psychiatric Assessment (PAPA) Journal of the American Academy of Child and Adolescent Psychiatry, 45(5), p. 538-549.

Egger, H., Ascher, B., \& Angold, A. (1999). Preschool Aged Psychiatric. AssessmentParent Interview. Unpublished manuscript, Center for Developmental Epidemiology, Department of Psychiatry and Behavioral Sciences, Duke University Medical Center, Durham, NC.

Eisen, M.L. (1997). The development and validation of a new measure of PTSD for Unpublished manuscript, California State University, Los Angeles.

Eisenberg N, Fabes R, Carlo G, Troyer D, Speer A, Karbon M, \& Switzer G. (1992). The relations of maternal practice and characteristics to children's vicarious emotional responsiveness. Child Development, 63(3), 583-602.

Fraiberg S. (Ed.). (1980). Clinical Studies in Infant Mental Health. New York: Basic Books.

Gunnar, M.R, \& Quevedo, K. (2007). The neurobiology of stress and development. Annu. Rev. Psychol. 58, p. 145-73.

Keenan, K., Shaw, D.S., Walsh, B., Delliquadri, E., Giovannelli, J. (1980). DSM-III-R Disorders in preschool children from low-income familiesx Journal of the American Academy of Child and Adolescent Psychiatry, 36(5), 620- 637. 
Laor, N., Wolmer, L., \& Cohen, DJ. (2001). Mothers functioning and children's symptoms in preschool-age children exposed to domestic violence. Journal of interpersonal Violence. 158(7), 1020 - 1036.

Lavigne, J.V, Arend, R., Rosenbaum, D., Binns, H.J., Christoffel, K.K., Gibbons, R.D. (1998). Psychiatric disorders with onset in the preschool years: I. Stability of diagnoses. Journal of the American Academy of Child and Adolescent Psychiatry. 37(12), $1246-1254$.

Lieberman, A.F., Van Horn, P., \& Ozer, E. (2005). Preschooler witnesses of marital violence: predictors and mediators of child behavior problems. Dev. Psychopathol. 17(2), $385-396$.

Linares, L. O, Heeren, T., Bronfman, E., Zuckerman, B., Augustyn, M., Tronick, E. (2001). A mediational model for the impact of exposure to community violence on early child behavior problems. Child Dev. 72(2), 639 - 652.

Lynch, M., \& Cicchetti, D. (1998). An ecological-transactional analysis of children and contexts: the longitudinal interplay among child maltreatment, community violence, and children's symptomatology. Dev. Psychopathol, 10(2), 235 - 257

Minde K, \& Minde R. (1986). Infant Psychiatry: An introductory textbook. Beverly Hills, CA: Sage.

Peebles C. (1997). The infant and preschool psychiatry clinic: A model of evaluation and treatment. Infant Mental Health Journal. 18(2) p. 221-229.

Pynoos, R., \& Nader, K. (1986). Witness to violence: Child interview. Journal of the American Academy of child Psychiatry, 25(3), 306-319. 
Num 20 - Julio-Diciembre, 2015 (Publicación preliminar)

Quota, S., Punamaki, R., El Sarraj. E (2003). Prevalence and determinants of PTSD among Palestinian Children Exponed to Military Violence. European Child and Adolescent Psychiatry, 12(6), p. 265-272.

Research Diagnostic Criteria -Preschool Age (RDC-PA). (August 17, 2002). (No publicado).

Rexford E, Sander L, Shapiro T. Infant Psychiatry. (1976). A New Synthesis. New Haven: Yale University Press.

Romero-Acosta, K. (2014). La comorbilidad de síntomas ansiosos y depresivos en la niñez. Nuevas directrices de investigación. Revista de Psicología Gepu, 5(2), p. 15 - 31.

Scheeringa, M. S., Zeanah, C.H., \& Myers, L. (2004). Heart period and variability findings in preschool children with posttraumatic stress symptoms. Biological Psychiatry, 55(7), p. 685-691.

Scheeringa, M. S., Zeanah, C. H., \& Myers, L. (2003). New findings on alternative criteria for PTSD in preschool children. Journal of the American Academy of Child \& Adolescent Psychiatry, 42(5), p. 561-570.

Scheeringa, MS., Zeanah, C.H., Myers, L., \& Putnam, F.W. (2005). Predictive validity in a prospective follow-up of PTSD in preschool children. Journal of the American Academy of Child and Adolescent Psychitry, 44(9), p. 889-906.

Scheeringa MS, Zeanah CH, Myers L, Putnam FW. (2002). Heart rate and RSA reactivity in traumatized preschool children. Symposium presented at the International Society for Traumatic Stress Studies 18th Annual Meeting, Baltimore, MD. 
Num 20 - Julio-Diciembre, 2015 (Publicación preliminar)

Scheeringa, M.S., Peebles, C.D., Cook, C.A., \& Zeanah,C.H. (2001). Toward Establishing procedural, criterion and discriminant validity for PTSD in early childhood. Journal of the American Academy of Child and Adolescent Psychiatry, 40(1), p. 52-60.

Scheeringa MS, Zeanah CH, Drell MJ, Larrieu JA. (1995). Two approaches to the diagnosis of posttraumatic stress disorder in infancy and early childhood. Journal of the American Academy of Child \& Adolescent Psychiatry, 34(2), p. 191-200.

Scheeringa, M.S., \& Zeanah, C.H. (1994). PTSD Semi-Structured interview and Observational Record gorInfants and Young Children. New Orleans, LA, Department of Psychiary and Neurology, Tulane University Health Sciences Center.

Stover C., Berkowits S. (2005). Assessing Violence Exposure and Trauma Symptom in Young Children: A Critical Review of Mesures. Journal of Traumatic Stress, 18(6), p. $7007-717$.

Stoddard FJ., Saxe G., Ronfeldt H., Drake JE., Burns J., Edgren C., Sheridan R. (2006). Acute Stress Symptoms in young children with burns. Journal of American Academy of Child and Adolescent Psychiatry. 45(1) p. 87-93.

Yule, W \& William, R., (1990). Posttraumatic Stress Reaction in Children. Journal of Traumatic Stress, 3(2), p. $279-295$.

World Health Organization. (1992). International Classification of Diseases and related health problems. Decimal version, Geneva. 